\title{
Photonics crystal fiber sensor using for sensing environmental pollution
}

\author{
Omar S.Hassan ${ }^{* 1}$, Bushra R.Mahdi ${ }^{* * 2}$, Razi J.Al-azawi ${ }^{* 3}$, Nahla A. Aljabar**.
}

\author{
*Department of laser and Optoelectronics, University of Technology, Baghdad, Iraqi \\ *1 ahm1296k@gmail.com ,*3 140009@uotechnology.edu.iq \\ ** Ministry of Science and Technology, Laser and Optoelectronic center, Iraq-Bagdad. \\ *2 Boshera65m@gmail.com.
}

Abstract: The aim of this paper is to create a sensor model based on optical crystal fibers (PCF). The aim of this model is to find and identify zinc cadmium. This thesis looked at three different categories of concentrations. For sensor architecture, PCF was generated using single-mode fiber-to-end split fusion (SM-PCF-SM). In this experiment, a specific wavelength spectrum was used to alter the concentration of materials covering the fiber in order to demonstrate the fiber's sensing capability. The $550 \mathrm{~nm}$ wavelength has been used as the optical source for the fiber. The change in the output power of the external light was monitored and changes were observed for each concentration of the concentrations around the fiber. It has been found that the fiber is sensitive to small changes in concentrations. The absorption of the fiber has been calculated for the incoming capacity, as well as the losses in the capacity outside the fiber.

Keywords: PCF; PCF sensor; fiber sensor; photonic crystal.

\section{Introduction}

Microstructured fibers (PCF) are a good alternative to traditional fibers with some special features, such as the transmission of radiant energy with decreased dispersion through the heart of the PCF [1], a high degree of non-linearity and a very low confinement losses (CL) [2][3].

The light propagation properties can now be monitored by changing the perimeter and the position of hole in both the middle and the retaining regions after the discovery of PCF. 3D printed dies and the sol-gel process are both possibilities to increase the air gaps [4]. Stacking and boiling are the two most common methods to change air trout in the middle of the PCF. [5]. High refractive index PCF's core area (RI). The largest electricity beam would therefore reach the central area of the PCF safely. Currently the identification of chemical and fluids by PCF is a general concern. To detect fluids and substances, it must be inserted into the core of the fiber [6]. As relative sensitivity (RS) depends in large proportion on the RI of the fiber, PCF needs a high level of RI. CL and resistance are the core properties of fluid and chemical sensing. Birefringence is an excellent PCF characteristic and is inherently desirable in high birefringence. Moreover, the CL and Effective material loss (EML) and efficient location are all effective area (EA).

During the last few years, a considerable number of geometric models based on PCFs have been proposed to boost the relative sensitivity, lower the CL, and raise the EA to an appropriate standard. a PCF sensor model was suggested by Sultana et al., to detect alcohol in 2018 [7], In the last few years, a large number of geometric PCF models to increase relative sensitivity have been proposed, reduce to an appropriate degree the CL and raise the EA. In 2018 Sultana et al. suggested a PCF alcohol detector sensor concept. [8]. In the same year, Islam et al. published another study using the $1.8 \mathrm{THz}$ frequency to detect poisonous chemicals. They got a 77.5 percent RS with an EML of $0.031 \mathrm{~cm}^{-1}$ and a confinement losses near the 5.1510-8 $\mathrm{cm}^{-1}$ and an [9]. 


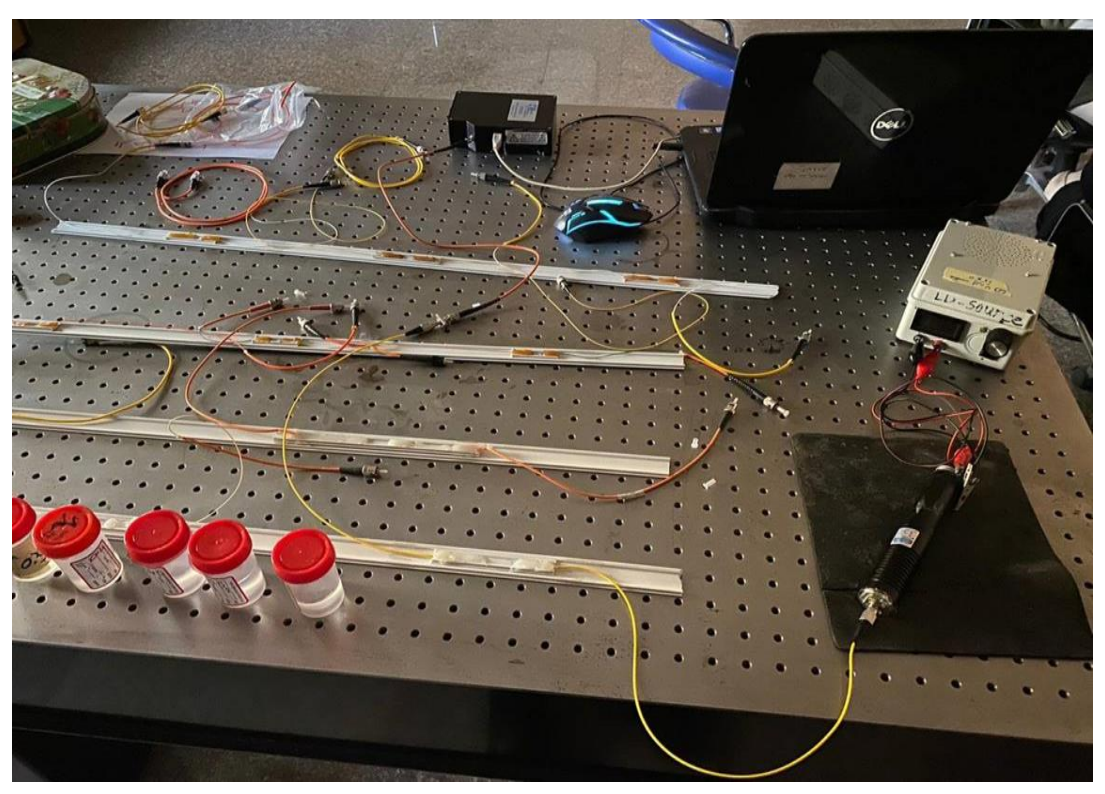

Figure 1. The setup of the experiment

\section{Design methodology}

We used the PCFs in our experiment (PCF LMA-10). It has a solid core surrounded by numbers of rings of air holes, with a core diameter of $10.6 \mu \mathrm{m}$, an air hole diameter of $3.08 \mu \mathrm{m}$, and a hole to hole spacing of $7.5 \mu \mathrm{m}$. To make this sensor, $2 \mathrm{~cm}$ of PCF was spliced between two SMF using a traditional fusion splicer. Until fusion splicing, the SMFs and PCFs are stripped of their silicone coatings and cleaved using a mechanical cleaver. PCF's air holes totally collapsed at the spliced stage. The collapsed area is about $300 \mu \mathrm{m}$ long in all [10][11]. As seen in Fig. 2, we consider the configuration of a refractive index optical

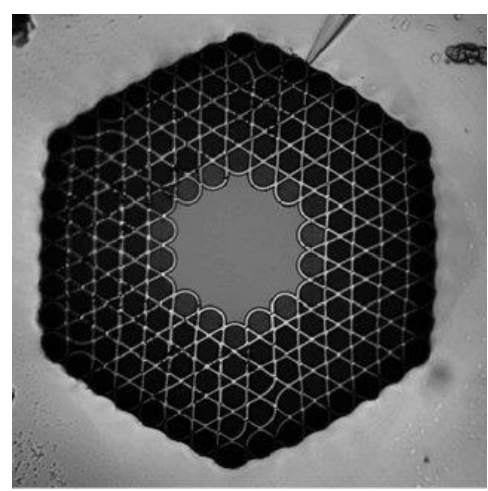

sensor made up of a PCF fiber with a wide mode area profile.

The fiber consists of a refractive index nucleus of glass surrounded by a collection of dielectric structures of refractive index 1 , resulting in the hole in the PCF fiber. The equation of the Sellmier's serves to calculate the index refractive [12]. It is known that the analytic medium to be analyzed fills the area beyond the fiber and has an external refractive index. D-shape cladding denotes the distance between the fiber's core and the met material layer, dam denotes the width of the met material layer, dhole denotes the depth of the holes, and K pitch refers to hollow separation. The PCF fiber profile prefers light containment in the nucleus, while the D-shape profile stimulates interaction between the

Figure 2. Cross section image of the the PCF. 
core modes and the external medium. A metal film that includes Plasmon surface mediates the combination of electromagnetic modes with outside media and increases sensitivity of the sensor. [10].

\section{Experimental}

In this part a description of the manufacturing process of the crystal optical fiber sensor is designed. This type of sensor is made by taking a piece of crystal fiber $2 \mathrm{~cm}$ long after removing the outer protective layer from both sides and cleaning it well with ethanol to ensure that the pieces of glass do not penetrate into the air openings.

The photonic crystal fiber piece is fusion spliced to a single-mode optical fiber on both sides, thus forming a sensor (SM-PCF-SM). Single mode optical fiber the outer protective layer is removed and cut with a single cutting machine to obtain a bound end to facilitate the welding process between the fiber pieces.

\section{Results}

As previously said, a laser source (532nm wavelength, $50 \mathrm{mw}$ power) is used, which is selected based on the zinc absorption spectrum. This experiment used PCF (LMA-10) with a $1.5 \mathrm{~cm}$ duration. The transmitted amplitude of this laser is seen in the figure below without any zinc concentration sample:

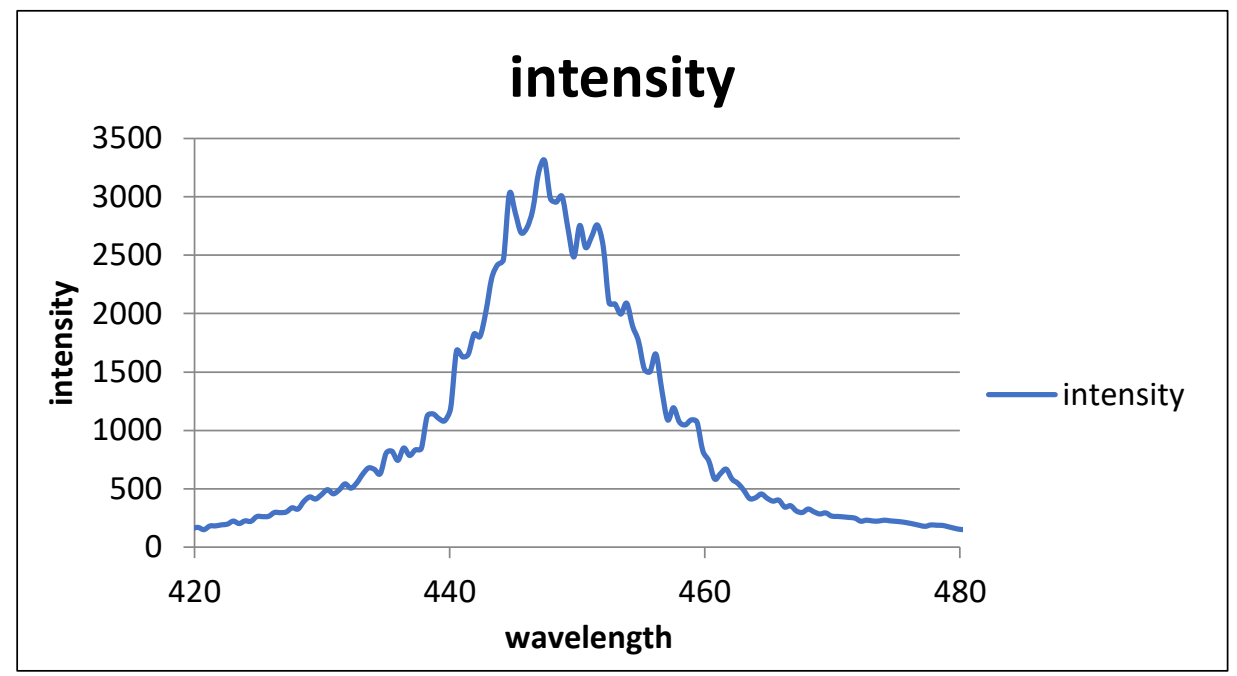

Figure 3. The green laser's output spectrum (532nm) without any external effects.

Until fully immerse the detector in the material sample, the maximum output reference intensity was 3312. Zinc Cadmium samples were collected in a variety of concentrations of the materials. Each concentration of zinc concentrate was determined using laser output spectroscopy. Tests of varying quantities of materials are analyses based on variations in the propagation laser density modulation. 


\section{Intensity of light in different concentration of Zn_Cd}

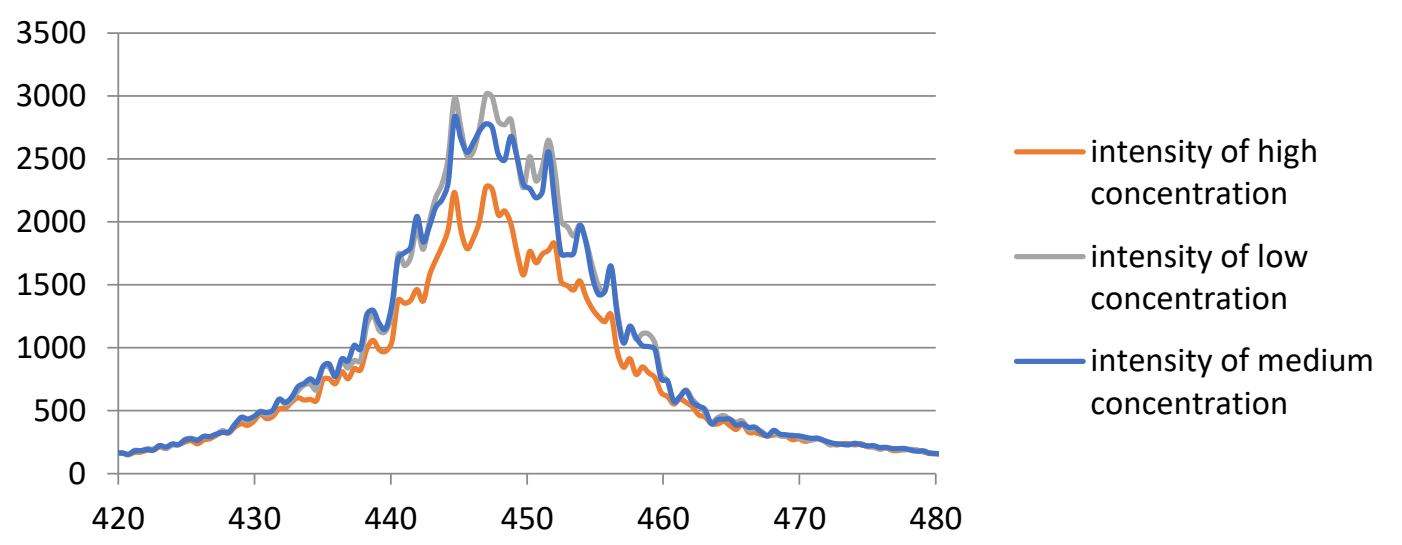

Figure 4. Laser sensor transmission spectra for Zn_Cd samples at 1.5cm LMA-10 PCF

The refractive indices and absorbance of each blood samples which is measured according to the reference intensity and the maximum intensity are tabulated in Table 1.

\begin{tabular}{|l|l|l|l|}
\hline samples & concentrations & $\begin{array}{l}\text { Maximum intensity } \\
\mathrm{I}_{0}\end{array}$ & $\begin{array}{l}\text { Absorbance } \\
\mathrm{A}=-\log \left(\mathrm{I}_{\mathrm{ref}} / \mathrm{I}_{0}\right)\end{array}$ \\
\hline high concentration & $\mathrm{zn}=10 \%: \mathrm{Cd}=10 \%$ & 2275 & 0.163108927 \\
\hline $\begin{array}{l}\text { medium } \\
\text { concentration }\end{array}$ & $\mathrm{zn}=1 \%: \mathrm{Cd}=8 \%$ & 2829 & 0.068457381 \\
\hline low concentration & $\mathrm{zn}=1 \%: \mathrm{Cd}=1 \%$ & 2929 & 0.053370956 \\
\hline
\end{tabular}

Table 1: Show the sensor properties.

Taking into account a spectral difference that can be reliably experimentally detected and using Eq. (1), the refractive index sensor spectral spectrum values for the metered content are indicated in Table 1. The similar findings for a plain metal layer sensor are also presented. In particular for a difference in the refractive index within the field of $[1.04,1.12]$ the sensor will actually work at $556 \mathrm{~nm}$, as predicted from Fig 4. 


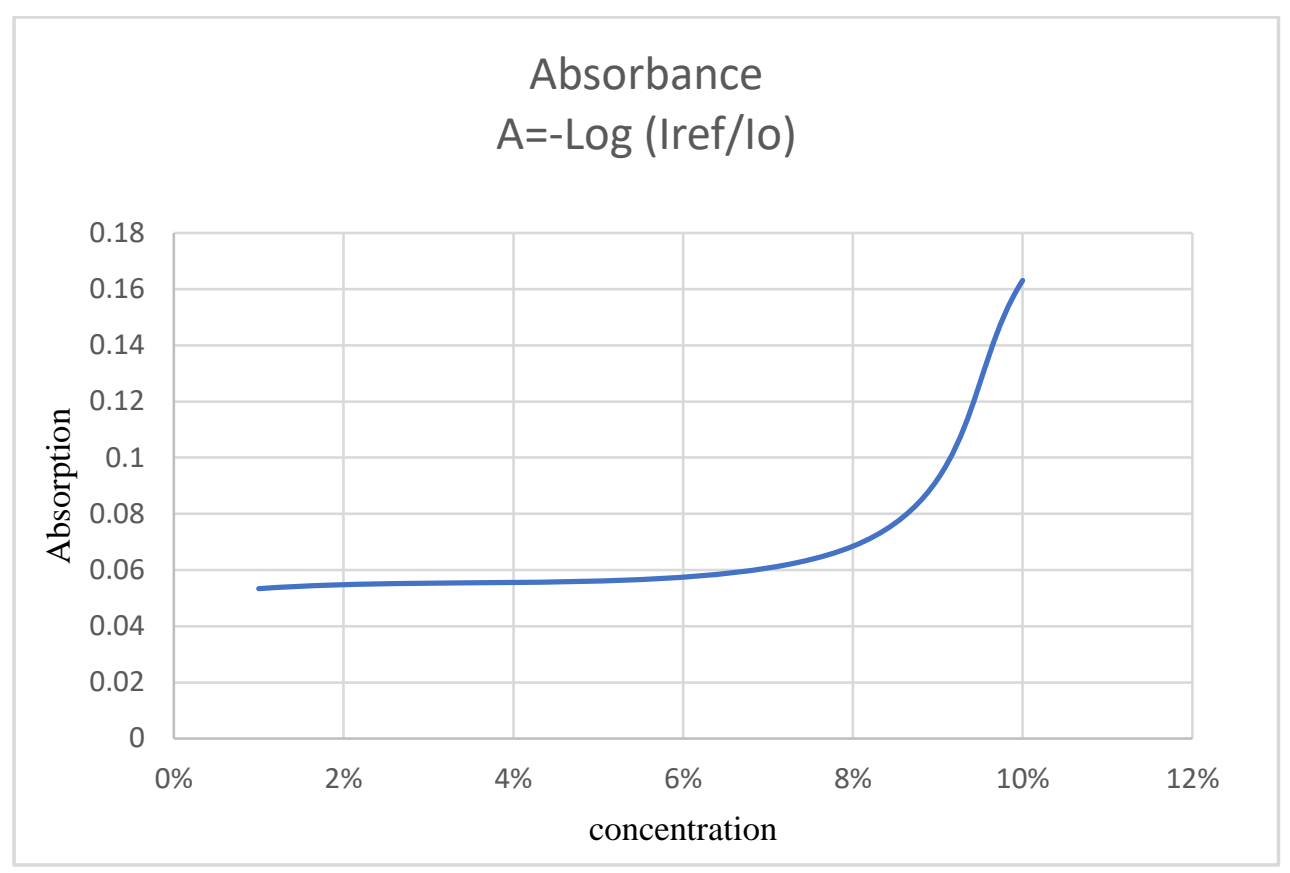

Figure 5. The relation between the absorption and concentration at $1.5 \mathrm{~cm}$ LMA-10 PCF

$\mathrm{A}=-\log \left(I_{\text {ref }} / I_{\mathrm{o}}\right)$

$I_{\text {ref }}$ refers the reference intensity or the intensity of the laser without any outside effect, $I_{\mathrm{o}}$ is the output intensity of the laser of the PCF surrounded with different concentration.

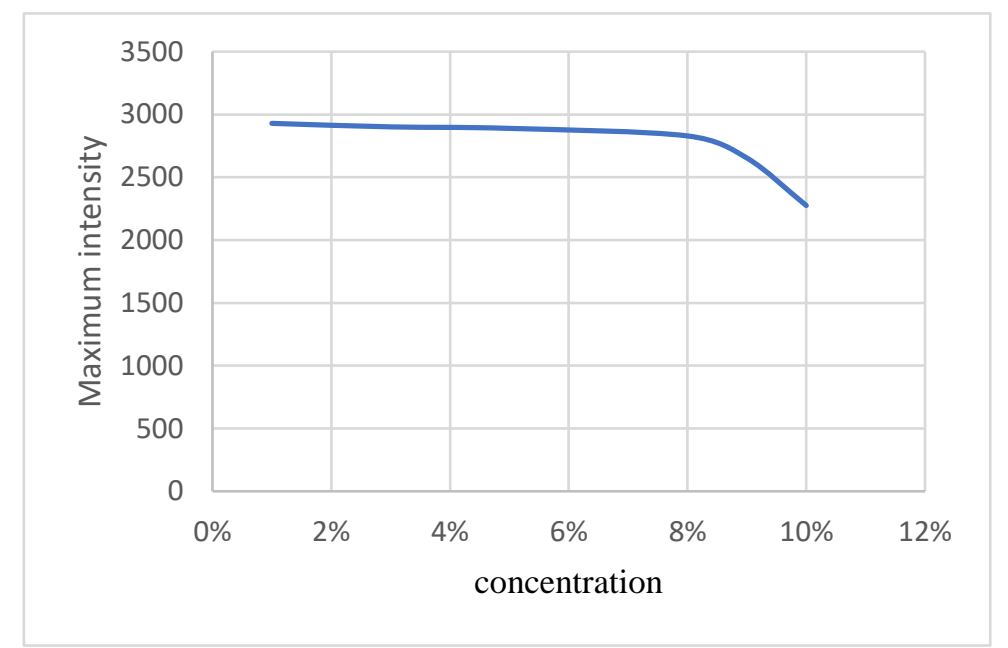

Figure 6. The relation between the maximum intensity with different concentration of Zn_Cd. 


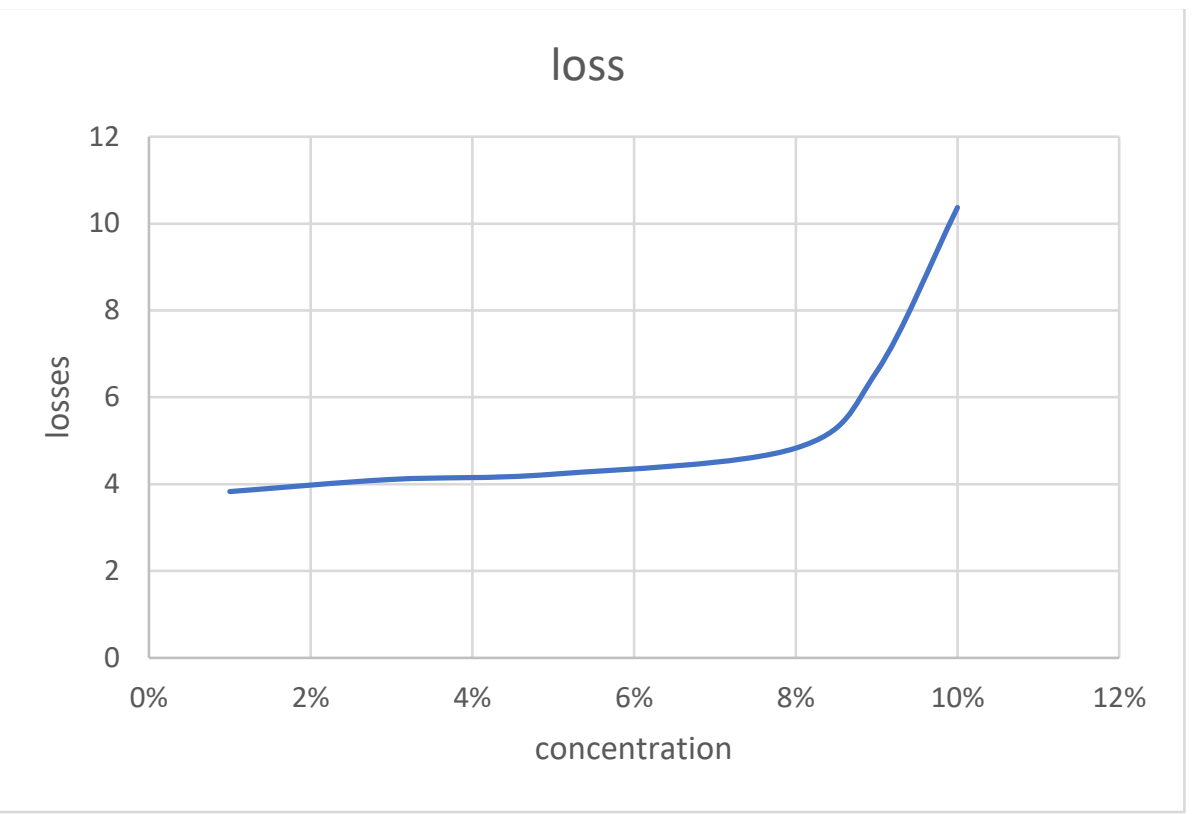

Figure 7. losses of the fiber with different concentration of $\mathrm{Zn} \_\mathrm{Cd}$.

\section{Conclusions}

In this research, we presented a new optical fiber sensor to detect the presence of fiber surrounding materials. The physical principle is based on the detection of materials surrounding the fiber to cause changes at the end of the optical fiber in terms of the intensity of radiation or the capacity of the outside energy, which reduces the reflection from the end of the fiber. The sensing concept is verified by means of experiments. The experimental results indicate that the sensor can measure certain samples of different concentrations of materials near or around the perimeter of the fiber crystal. The capacity of the fiber has been measured for several concentrations and the amount of loss measured for a certain range of concentrations.

\section{References}

[1] Ayad Z. mohammed, "Photonic crystal fiber Mach-Zehnder interferometer pH sensing," AIP Conf. Proc., vol. 2045, pp. 1-6, 2018, doi: 10.1063/1.5080823.

[2] S. R. Tahhan and H. K. Aljobouri, “Sensing of Illegal Drugs by Using Photonic Crystal Fiber in Terahertz Regime," 2020.

[3] Hiyam S. Ali,a, Makram A. Fakhri, "An overview of Au \& photonic crystal fiber of sensors," Mater. Sci. Forum, vol. 1002, no. Figure 1, pp. 282-289, 2020, doi: 10.4028/www.scientific.net/MSF.1002.282.

[4] S. R. Tahhan, A. J. Ghazai, I. M. Alwan, and M. H. Ali, “INVESTIGATION OF THE CHARACTERISTICS OF HIGH-RESISTIVITY SILICA BASED HYBRID POROUS CORE PHOTONIC CRYSTAL FIBER FOR TERAHERTZ WAVE GUIDANCE," vol. 14, no. 3, pp. 831-841, 2019.

[5] F. Iqbal, S. Biswas, A. A. Bulbul, and H. Rahaman, "Alcohol Sensing and Classification using PCF-based Sensor," Sens. BioSensing Res., vol. 20, 2020, doi: 10.1016/j.sbsr.2020.100384.

[6] B. R. M. Farah H. Hamza, Ayad Z. Mohammed, "Construction of optical fiber humidity sensor by laser technique," AIP Conf. Proc., vol. 2213, no. March, 2020, doi: 10.1063/5.0000337.

[7] J. A. S. Ultana and M. D. S. A. I. Slam, “Terahertz detection of alcohol using a photonic crystal fiber sensor," vol. 57, no. 10, pp. 2426-2433, 2018.

[8] S. Rana, N. Kandadai, and H. Subbaraman, "A Highly Sensitive, Polarization Maintaining Photonic Crystal Fiber Sensor Operating in the THz Regime," 2018, doi: 10.3390/photonics5040040.

[9] S. Islam, J. Sultana, A. Dinovitser, K. Ahmed, B. W. Ng, and D. Abbott, "Sensing of toxic chemicals using polarized photonic crystal fiber in the terahertz regime," vol. 426, no. May, pp. 341-347, 2018.

[10] B. R. Mahdi, L. K. Humdi, and L. S. Branch, "PHOTONIC CRYSTAL FIBER MAGNETIC FIELD SENSOR BASED ON," vol. 29, no. 1, pp. 99-102, 2017.

[11] R. Hani, B. R. Mahdi, and A. Z. Mohammed, "Photonic crystal fiber sensor for blood with different concentration of zinc," Mater. Sci. Forum, vol. 1002, pp. 290-299, 2020, doi: 10.4028/www.scientific.net/MSF.1002.290. 
[12] C. H. Huang, G. Zhang, Z. Q. Chen, X. J. Huang, and H. Y. Shen, “Calculation of the absorption coe cients of optical materials by measuring the transmissivities and refractive indices," vol. 34, pp. 209-211, 2002. 